\title{
Nominata de avaliadores do ano de 2014
}

A Direção da RAP agradece aos professores e pesquisadores que vêm colaborando para o aprimoramento da qualidade do conteúdo dos trabalhos divulgados pela revista. Sem dúvida alguma, a RAP deve, em grande parte, a sua posição como um dos principais periódicos na área de administração pública/administração ao trabalho voluntário desses avaliadores.

Adelaide Maria Coelho BâtTa

Centro Universitário de Sete Lagoas (Unifemm)

AdELAR FochezATto

Pontifícia Universidade Católica do Rio Grande do Sul (PUC-RS)

AdEMar DUtra

Universidade do Sul de Santa Catarina (Unisul)

Alberto Luiz Albertin

Fundação Getulio Vargas (FGV/Eaesp)

Alexandre de PÁdua Carrieri

Universidade Federal de Minas Gerais (UFMG)

Alessandra de Sá Mello da Costa

Pontifícia Universidade Católica do Rio de Janeiro (PUC-RJ)

Aguinaldo Aragon Fernandes

Instituto de Tecnologia Aragon \& Costa

Américo da Costa Ramos Filho

Universidade Federal Rural do Rio de Janeiro (UFRRJ)

Ana Carolina Pimentel Duarte da Fonseca

Universidade Federal do Rio de Janeiro (UFRJ)

Ana Cristina Brito Arcoverde

Universidade Federal de Pernambuco (UFPE) 
Ana Elisa PÉRICo

Universidade Estadual Paulista (Unesp)

Ana Luiza d'Ávila Viana

Universidade de São Paulo (USP)

Ana Luísa Vieira de Azevedo

Centro Universitário Unicarioca

Ana Maria de Albuquerque Vasconcellos

Universidade da Amazônia (Unama)

André Luiz Marques Serrano

Universidade de Brasília (UnB)

ANDRÉ Pimenta Freire

Universidade Federal de Lavras (UFLA)

ANDRei KoERner

Universidade Estadual de Campinas (Unicamp)

AnElise Graciele Rambo

Universidade Federal da Fronteira Sul (UFFS)

Antonio Artur de SouzA

Universidade Federal de Minas Gerais (UFMG)

Antonio GonçAlves Oliveira

Universidade Tecnológica Federal do Paraná (UTFPR)

Antonio Roberto Bono Olenscki

Universidade Estadual Paulista (Unesp)

ARILDA SCHMIDT Godoy

Universidade Presbiteriana Mackenzie

Biancca Scarpeline de CASTRO

Universidade Federal Rural do Rio de Janeiro (UFRRJ)

Camila Potyara Pereira

Universidade de Brasília (UnB) 
CARLA Bronzo

Fundação João Pinheiro (FJP)

Carlos Alberto de Vasconcelos Rocha

PUC-MG

CARLOS R. ETULAin

Universidade Estadual de Campinas (Unicamp)

Carlos Eduardo Gasparini

Universidade Castelo Branco

Carmen Nila Phang Romero Casas

Fundação Oswaldo Cruz (Fiocruz)

CARMen Rosario Ortiz Gutierrez GeLINSKi

Universidade Federal de Santa Catarina (UFSC)

Cezarina Maria Nobre Souza

Instituto Federal de Educação, Ciência e Tecnologia do Pará (IFPA)

Claudia Risso de Araujo Lima

Departamento de Informática do SUS (Datasus)

Debora Gomes Machado

Fundação Universidade Federal do Rio Grande (Furg) e Universidade Regional de Blumenau (Furb)

Eduardo CERQueira Batitucci

Fundação João Pinheiro (FJP)

EduARDo de Lima CALDAS

Universidade de São Paulo (USP)

Eduardo Granha Magalhães Gomes

Ministério da Educação (MEC)

Eduardo Henrique Diniz

Fundação Getulio Vargas (FGV/Eaesp) 
Elaine Rabelo NeIVA

Universidade de Brasília (UnB)

EnRique Pastor SEller

Universidad de Murcia - Espanha

ERnesto Fernando R. Vicente

Universidade Federal de Santa Catarina (UFSC)

FABIANO MAURY RAUPP

Universidade do Estado de Santa Catarina (Udesc)

FÁBIO GuEdes Gomes

Universidade Federal de Alagoas (UFAL)

FABIO KLEIN

Escola de Artes, Ciências e Humanidades da Universidade de São Paulo (curso de Gestão de Políticas Públicas - USP)

FÁtima BAYMa De Oliveira

Fundação Getulio Vargas (FGV/ Ebape)

Fernanda Filgueiras Sauerbronn

Universidade Federal do Rio de Janeiro (UFRJ)

Fernando Bastos Costas

Universidade Federal do Rio Grande do Norte (UFRN)

Fernando de Souza Coelho

Universidade de São Paulo (USP)

FERnANDo Dewes

Universidade de Caxias do Sul (UCS)

FERNANDO FACURY SCAFF

Universidade de São Paulo (USP)

Florence Cavalcanti Heber Pedreira

Universidade Federal de Sergipe (UFS)

Frederico Augusto Barbosa da Silva

Instituto de Pesquisa Econômica Aplicada (Ipea) 
Gabriela Brelaz

Universidade Federal de São Paulo (Unifesp)

Gilda Cardoso Araujo

Universidade Federal do Espírito Santo (Ufes)

Gilmar Ribeiro de Mello

Universidade de São Paulo (USP)

Gislene de Fátima Pereira

Universidade Federal do Paraná (UFPR)

IVAn AntôNio Pinheiro

Universidade Federal do Rio Grande do Sul (UFRGS)

IVAN RICARDo GARTNER

Universidade de Brasília (UnB)

Jackeline Amantino de Andrade

Universidade Federal de Pernambuco (UFPE)

JAN Bitoun

Universidade Federal de Pernambuco (UFPE)

JanAÍNA Machado SimÕES

Universidade Federal Rural do Rio de Janeiro (UFRRJ)

JANANN Joslin Medeiros

Universidade de Brasília (UnB)

JEROEN JOHANNES KLINK

Universidade Federal do ABC (UFABC)

João Batista Pamplona

Pontifícia Universidade Católica de São Paulo (PUC-SP)

JoÃo Henrique Gurtler Scatena

Universidade Federal de Mato Grosso (UFMT)

José ÂNGElo Machado

Universidade Federal de Minas Gerais (UFMG) 
José Antonio Gomes de Pinho

Universidade Federal da Bahia (Ufba)

José Augusto Abreu SÁ Fortes

Universidade de Brasília (UnB)

José CARLOS BARBIERI

FGV/EAESP

José Elias Feres de AlmeidA

Universidade Federal do Espírito Santo (Ufes)

José LuIz AlVEs

Universidade de Pernambuco (UPE)

José Matias Pereira

Universidade de Brasília (UnB)

JosÉ Osvaldo De SoRdi

Universidade Nove de Julho (Uninove)

José Paulo Cosenza

Universidade Federal Fluminense (UFF)

José Roberto R. Afonso

Fundação Getulio Vargas (FGV/ Ibre)

Kely Cesar Martins de Paiva

Universidade Federal de Minas Gerais (UFMG)

Klitia Valeska Bicalho de Sá

Universidade Federal Rural do Rio de Janeiro (UFRRJ)

KuRt Eberhart VON MetTEnHeIM

Fundação Getulio Vargas (FGV/Eaesp)

LETICIA Dias FANTINEL

Universidade Federal da Bahia (Ufba)

Lucia Maria Machado Bogus

Pontifícia Universidade Católica de São Paulo (PUC-SP) 
LuCiana Gross CunHA

Fundação Getulio Vargas (FGV/Eaesp)

LUCIANO Rossoni

Universidade do Grande Rio (UNIGRANRIO)

LUCIO BITTENCOURT

Fundação Getulio Vargas (FGV/Eaesp)

Luis Antonio BARone

Universidade Estadual Paulista (Unesp)

LUIS RoQue KLERING

Universidade Federal do Rio Grande do Sul (UFRGS)

Marcello Beckert Zappellini

Universidade do Estado de Santa Catarina (UDESC)

Marcia Regina Ferreira

Universidade Federal do Paraná (UFPA)

Marcia da Silva Mazon

Universidade Federal de Santa Catarina (UFSC)

Marcilene Garcia de Souza

Universidade Estadual Paulista (Unesp)

Marco Antonio Carvalho Teixeira

Fundação Getulio Vargas (FGV/Eaesp)

Marcus Vinícius Peinado Gomes

Fundação Getulio Vargas (FGV/Eaesp)

Maria Carolina Martinez Andion

Universidade do Estado de Santa Catarina (Udesc)

Maria Ceci Araujo Misoczky

Universidade Federal do Rio Grande do Sul (UFRGS)

Maria Ester de Freitas

Fundação Getulio Vargas (FGV/Eaesp) 
Maria Gracinda Carvalho Teixeira

Universidade Federal Rural do Rio de Janeiro (UFRRJ)

Maria Inês CaEtano FerReira

Universidade Federal do Recôncavo da Bahia (UFRB)

Maria Tereza Sadek

Universidade de São Paulo (USP)

Mario Cesar Feereira

Universidade de Brasília (UnB)

Marília Patta Ramos

Universidade Federal do Rio Grande do Sul (UFRGS)

Mario Roberto Dal Poz

Universidade do Estado do Rio de Janeiro (Uerj)

Marlene Catarina de Oliveira Lopes Melo

Faculdade Novos Horizontes

Marli Elizabeth RitTer dos Santos

Pontifícia Universidade Católica do Rio Grande do Sul (PUC-RS)

Marta Ferreira Santos Farah

Fundação Getulio Vargas (FGV/Eaesp)

Miriam RodRigues

Universidade Presbiteriana Mackenzie

Monaliza Oliveira Ferreira

Universidade Federal de Pernambuco (UFPE)

NicOlau REINHARD

Universidade de São Paulo (USP)

ORENZIO SOLER

Universidade Federal do Rio de Janeiro (UFRJ)

OtÁvio Prado

Fundação Getulio Vargas (FGV/Eaesp) 
Paloma Maria Santos

Universidade Federal de Santa Catarina (UFSC)

Patrícia Almeida Ashley

Universidade Federal Fluminense (UFF)

PATRÍCIA LACZYNSKI

Fundação Getulio Vargas (CEAPG — FGV/Eaesp)

Patricia Maria Emerenciano de Mendonça

Fundação Educacional Inaciana (FEI)

Paula Chies Schommer

Universidade Federal da Bahia (Ufba)

Paulo Carlos Du Pin Calmon

Universidade de Brasília (UnB)

Pedro Aguerre

Pontifícia Universidade Católica de São Paulo (PUC-SP)

Pedro Bendassolli

Universidade Federal do Rio Grande do Norte (UFRN)

Reginaldo Souza Santos

Universidade Federal da Bahia (Ufba)

Reinaldo Santos Andrade

Universidade Estadual de Feira de Santana (Uefs)

ReNATO CADER

Instituto de Pesquisa Jardim Botânico

RENATO SÉRgio DE LiMA

Fundação Seade

Ricardo Borges Gama Neto

Universidade Federal de Pernambuco (UFPE)

RicARDo CoRrea Gomes

Universidade de Brasília (UnB)

Ricardo Vieira de Carvalho Fernandes

Universidade de Brasília (UnB) 
Roberto de Souza Rodrigues

Universidade Federal Rural do Rio de Janeiro (UFRRJ)

Roberto de Sousa Miranda

Universidade Federal de Campina Grande (UFCG)

Rosa Maria Marques

Pontifícia Universidade Católica de São Paulo (PUC-SP)

Rosana Rodrigues Heringer

Universidade Federal do Rio de Janeiro (UFRJ)

Rosinha da Silva Machado Carrion

Universidade Federal do Rio Grande do Sul (UFRGS)

RUTHY NADIA LANIADO

Universidade Federal da Bahia (Ufba)

SABRina SoARES da SiLVA

Universidade Federal de Lavras (Ufla)

Santiago Falluh Varella

Conselho Nacional de Justiça

Sergio Luiz Moraes Pinto

Fundação Getulio Vargas (FGV/Eaesp)

SONIA MaRia Dias

Mulheres no Emprego Informal: Globalizando e Organizando (Wiego)

Sonia Maria Fleury Teixeira

Fundação Getulio Vargas (FGV/Ebape)

Sonia Valle Walter Borges de Oliveira

Universidade de São Paulo (USP)

Teresa Cristina de Oliveira Nunes

Universidade Federal de Juiz de Fora (UFJF)

Terezinha Noemides Pires Alves

Universidade Federal de Juiz de Fora (UFJF)

ThIERry Molnar Prates

Universidade Estadual de Ponta Grossa (UEPG) 
Tiago Wickstrom Alves

Universidade do Vale do Rio dos Sinos (Unisinos)

Tomas de Aquino Guimarães

Universidade de Brasília (UnB)

URsula Dias Peres

Universidade de São Paulo (USP)

Valderez Ferreira Fraga

Fundação Getulio Vargas (FGV/Ebape)

Valdete Maria Ruiz

Centro Universitário das Faculdades Associadas de Ensino (Unifae)

Valéria Gama Fully Bressan

Universidade Federal de Minas Gerais (UFMG)

VANEssa Elias de OliveIRA

Universidade Federal do ABC (UFABC)

VANESSA S. ChAER Kishima

Fundação Getulio Vargas (FGV/Eaesp)

Victor MeYer

Pontifícia Universidade Católica do Paraná (PUC-PR)

WAgner Costa Ribeiro

Universidade de São Paulo (USP)

ZÉLIA MiRANDA KILIMNIK

Universidade Federal de Minas Gerais (UFMG) e Universidade Fumec

Zilma Borges DE SouzA

Fundação Getulio Vargas (FGV/Eaesp) 